\title{
KINETICS OF CHARGE TRANSFER PROCESSES IN MOLECULAR JUNCTIONS
}

\author{
E.G. PETROV
}

PACS 87.15.-v, 85.65. + h,

Bogolyubov Institute for Theoretical Physics, Nat. Acad. of Sci. of Ukraine

(C) 2011

(14b, Metrologichna Str., Kyiv 03680, Ukraine)

\begin{abstract}
A kinetic master equation for state populations of a quantum system comprised of separate quantum subsystems, is derived. The equation allows one to describe the charge transfer processes in molecular junctions, where the molecule operates as a transmitter of electrons between the electrodes. Special attention is given to the derivation of contact and distant rate constants responsible for the formation of sequential (hopping) and direct (distant) components of the current, as well as for the time evolution of molecular state probabilities.
\end{abstract}

\section{Introduction}

In last two decades, the studies of charge transfer processes in molecular devices like "electrode 1-moleculeelectrode 2" (1M2) have aroused a great interest [1-8]. This is dictated by a possibility to utilize the organic molecules as basic elements of electronics. The theoretical description of the charge transfer in 1M2-devices is mainly analyzed in the model, where a molecule embedded between two leads acts as a dynamic scattering center. Respective description is based on the Landauer's approach with the use of the nonequilibrium Green function method in combination with quantum-mechanical calculations. This allows one to derive the expressions quite suitable for a numerical estimation of the current through a molecule (see, for instance, $[3,7,9]$ ). The Landauer's approach gives a rather good description of experimental results if only the multiparticle effects are minor during the charge transmission [10].

Another approach is based on the nonequilibrium density matrix method [11-19]. Adaptations of this method to the description of charge-transfer processes in molecular devices can be found in [20-26], where the device is considered as an open quantum system, while the macroscopic electrodes and the environmental vibrations refer to the electron/hole and phonon reservoirs, respectively. The method offers a unified description of the tunneling and hopping charge transfer processes with regard for the Coulomb repulsion between the electrons captured by a molecule in the course of their transmission through a molecule $[22,24,25,27-29]$. The unified description is based on the reduction of the generalized master equation for the state populations of the whole transfer system to the kinetic equations for the states belonging to separate parts of the system. In the case of macroscopic electrodes and the fast vibrational relaxation within a molecule, the noted kinetic equations appear in the form of linear balance-like equations for populations of the electrode's electrons and state populations of a neutral or charged molecule. The respective transfer rates characterize both the contact (electrodemolecule) and distant (electrode-electrode) charge transitions, as well as the intramolecular site-to-site transitions. The form of transfer rates has been derived for different particular cases.

In this paper, we derive a general form for both an inter-electrode current and kinetic equations related to molecular state populations. Simultaneously, the expressions for the respective contact and distant transfer rates are given. These expressions are used to calculate the rate constants characterizing the different types of transmission processes in a $1 \mathrm{M} 2$-device.

\section{Basic Master Equation for State Populations of a Quantum System}

A rigorous description of the dynamics of a quantum system (QS) is achieved with the Liouville equation for the QS density matrix $\rho_{\mathrm{QS}}(t)$,

$\dot{\rho}_{\mathrm{QS}}(t)=-\mathrm{i} \mathcal{L}(t) \rho_{\mathrm{QS}}(t)$,

where $\mathcal{L}(t)=(1 / \hbar)\left[H_{\mathrm{QS}}(t), \ldots\right]$ is the Liouville superoperator associated with the Hamiltonian

$H_{\mathrm{QS}}(t)=H_{0}+\Delta H(t)+V(t)$.

Here,

$H_{0}=\sum_{a} E(a)|a\rangle\langle a|$

is the main part of the QS Hamiltonian with $E(a)$ and $|a\rangle$ being its proper energy and proper state, respectively. 
Transitions between the QS states are determined by the off-diagonal interaction

$V(t)=\sum_{a, b} V_{a b}(t)\left(1-\delta_{a, b}\right)|a\rangle\langle b|$

Operator (4) depends on time $t$ if the transitions between the QS states are caused not only by stationary (e.g., electron-vibrational) interactions but external or/and internal (fluctuation) fields. The term

$\Delta H(t)=\sum_{a} \Delta E_{a}(t)|a\rangle\langle a|$

characterizes the action of external regular or/and stochastic fields shifting the energy levels in such a manner that the QS energies $E_{a}$ gain respective additions $\Delta E_{a}(t)$.

\subsection{Exact master equations for diagonal and off-diagonal parts of the $Q S$ density matrix}

Using the method of projection operators $[11,12,22,24$, $30]$, one can derive a system of coupled differential equations for the diagonal, $\rho_{d}(t)=\sum_{a}\left\langle a\left|\rho_{\mathrm{QS}}(t)\right| a\right\rangle|a\rangle\langle a| \equiv$ $\hat{T}_{d} \rho_{\mathrm{QS}}(t)$, and off-diagonal, $\rho_{n d}(t)=\sum_{a, b}(1-$ $\left.\delta_{a b}\right)\left\langle a\left|\rho_{\mathrm{QS}}(t)\right| b\right\rangle|a\rangle\langle b| \equiv \hat{T}_{n d} \rho_{\mathrm{QS}}(t)$, parts of the density matrix. These equations read

$\dot{\rho}_{d}(t)=-\mathrm{i} \hat{T}_{d} \mathcal{L}_{V}(t) \rho_{n d}(t)$

$\dot{\rho}_{n d}(t)=-\mathrm{i} \hat{T}_{n d}\left(\mathcal{L}_{0}(t)+\mathcal{L}_{V}(t)\right) \rho_{n d}(t)-\mathrm{i} \mathcal{L}_{V}(t) \rho_{d}(t)$,

where $\mathcal{L}_{0}(t)=(1 / \hbar)\left[H_{0}+\Delta H(t), \ldots\right]$ and $\mathcal{L}_{V}(t)=$ $(1 / \hbar)[V(t), \ldots]$.

After the substitution of one equation in the other, the following master equations are obtained for the diagonal and off-diagonal parts of the density matrix:

$\dot{\rho}_{d}(t)=-\int_{0}^{t} d \tau \hat{T}_{d} \mathcal{L}_{V}(t) S(t) S^{+}(t-\tau) \mathcal{L}_{V}(t-\tau) \rho_{d}(t-\tau)$

and

$$
\begin{aligned}
& \dot{\rho}_{n d}(t)=-\mathrm{i} \hat{T}_{n d}\left(\mathcal{L}_{0}(t)+\mathcal{L}_{V}(t)\right) \rho_{n d}(t)- \\
& -\mathcal{L}_{V}(t) \hat{T}_{d} \int_{0}^{t} d \tau \mathcal{L}_{V}(t-\tau) \rho_{n d}(t-\tau),
\end{aligned}
$$

respectively. In Eq. (7),

$$
S(t)=\hat{P} \mathrm{e}^{-\mathrm{i} \int_{0}^{t} d \tau^{\prime}\left[\mathcal{L}_{0}\left(\tau^{\prime}\right)+\hat{T}_{n d} \mathcal{L}_{V}\left(\tau^{\prime}\right)\right]}
$$

is the evolution superoperator ( $\hat{P}$ is the Dyson's chronological operator). The master equations (7) and (8) have been derived without use of any approximation in the interactions $V(t)$ and $\Delta H(t)$. Thus, these equations can be employed as the basic ones for the calculation of state populations $\mathcal{P}(a ; t)=\left\langle a\left|\rho_{d}(t)\right| a\right\rangle$ and coherences $\mathcal{Y}_{a b}(t)=\left\langle a\left|\rho_{n d}(t)\right| b\right\rangle$. In this paper, we deal only with state populations. With use of Eq. (7) and in line with the definition of the quantities $\mathcal{P}(a ; t)$ and $S(t)$, one derives the following exact master equation for the state occupancies:

$\dot{\mathcal{P}}(a ; t)=-\frac{1}{\hbar^{2}} \sum_{b(\neq a)} \int_{0}^{t} d \tau \mathcal{G}_{a b}(t, t-\tau)[\mathcal{P}(a ; t-\tau)-$

$-\mathcal{P}(b ; t-\tau)]$

where the kernel

$\mathcal{G}_{a b}(t, t-\tau)=\sum_{l l^{\prime}}\left[U_{a l}(t, t-\tau) V_{l b} U^{+}(t, t-\tau)_{b l^{\prime}} V_{l^{\prime} a}(t)-\right.$

$\left.-V_{a l}(t) U_{l l^{\prime}}(t, t-\tau) V_{l^{\prime} b}\left(U^{+}(t, t-\tau)\right)_{b a}+c . c.\right]$

is defined via the matrix elements $V_{l l^{\prime}}(t)=\left\langle l|V(t)| l^{\prime}\right\rangle$ and $U_{l l^{\prime}}(t, t-\tau)=\left\langle l\left|U(t, 0) U^{+}(t-\tau, 0)\right| l^{\prime}\right\rangle=\left(U^{+}(t, t-\right.$ $\tau))_{l^{\prime} l}$ with

$U(t, 0)=\hat{P} \mathrm{e}^{-(i / \hbar) \int_{0}^{t} d \tau^{\prime}\left[H_{0}+\Delta H\left(\tau^{\prime}\right)+\hat{T}_{n d} V\left(\tau^{\prime}\right)\right]}$

being the propagator. Note that the integro-differential equation (10) appears as a stochastic equation of the non-Markovian type if only the interactions $\Delta H(t)$ and $V(t)$ are stochastic operators.

\subsection{Coarse-grained master equation for $Q S$ state populations}

In numerous practically important applications, the description of transfer processes is carried out with much simpler balance-like kinetic equations of the Markovian type. There are different physical reasons for which the exact master equation (10) could be reduced to the noted equations. One of the reasons is that the transitions in a QS are caused by the time-independent off-diagonal interaction $V$ in the presence of a fast stochastic field 
shifting the QS energy levels in a random way. Just such a situation is considered in the present paper. It is supposed that a stochastic field is created by high frequency vibrations of a molecule, while the transitions between the QS states are accompanied by low-frequency vibrations (see the discussion in [31]).

Let the characteristic time of transition processes associated with the interaction $V$ be $\tau_{\mathrm{tr}}$, and let the characteristic time of random alterations of the energy positions be $\tau_{\text {stoch }}$. If the strong inequality

$\tau_{\text {stoch }} \ll \tau_{\text {tr }}$

is satisfied, then the averaging $\langle\langle\ldots\rangle\rangle$ of the master equation (10) over the random realizations of the fast stochastic field leads to the decoupling procedure $\left\langle\left\langle\mathcal{G}_{a b}(t, t-\right.\right.$ $\tau) \mathcal{P}(b ; t-\tau)\rangle\rangle \approx G_{a b}(\tau) P(b ; t-\tau)$, where $G_{a b}(\tau)=$ $\left\langle\left\langle\mathcal{G}_{a b}(t, t-\tau)\right\rangle\right\rangle$ and $P(b ; t-\tau)=\langle\langle\mathcal{P}(b ; t-\tau)\rangle\rangle$. An important result is that the averaged kernel, $G_{a b}(\tau)$, exhibits an exponential decrease with their own characteristic times $\tau_{\text {stoch }}$ (see the examples in [31-36]). Therefore, owing to condition (13), one can shift the upper limit in the integral from $t$ to infinity and set $P(b ; t-\tau) \approx P(b ; t)$. Thus, the averaging of the master equation (10) over the random realizations of the fast stochastic field reduces this equation to the coarsegrained kinetic equation

$\dot{P}(a ; t)=-\sum_{b(\neq a)} K(a ; b)[P(a ; t)-P(b ; t)]$

valid on the time scale $\Delta t \sim \tau_{\mathrm{tr}}$. In Eq. (14), the quantity $K(a ; b)=\left(1 / \hbar^{2}\right) \int_{0}^{\infty} d \tau G_{a b}(\tau)$ is expressed through the integral of the averaged kernel of the basic master equation (10). Equation (14) describes an evolution of averaged state occupancies $P(a ; t)$ that are coupled by the normalization condition

$\sum_{a} P(a ; t)=1$

Particular cases of the noted reduction procedure can be found in [31-36], where a stochastic field is given by different types of discrete processes including wellknown dichotomous and trichotomous random realizations. The simplest form for the transfer rate $K(a ; b)$ occurs if one ignores the broadening of the QS energy levels caused by a stochastic field. In this case, following the method offered in [30], one can represent the transfer rate in the form [37]

$K(a ; b)=\frac{2 \pi}{\hbar}\left|V_{b a}^{(t r)}\right|^{2} \delta[E(a)-E(b)]$ where $V_{b a}^{(\operatorname{tr})}=\left\langle b\left|V^{(\operatorname{tr})}\right| a\right\rangle$ is the transition matrix element on the energy surface $E=E_{a}=E_{b}$, and

$V^{(\operatorname{tr})}=V+V G(E) V$

is the transition operator. The latter contains the Green's operator $G(E)=\left(E-H+i 0^{+}\right)^{-1}$ with

$H=H_{0}+V$

being the QS Hamiltonian taken in the absence of a stochastic field [38]. Forms similar to that of Eq. (17) are widely employed for the description of scattering processes when initial, $|a\rangle$, and final, $|b\rangle$, states belong to the colliding particles with and without changes in their compositions [39]. Note, however, that each state in the scattering theory includes necessarily states of the continuous spectrum of the particle. In our case, the presence of the continuous spectrum is replaced by the random shifts of the QS energy levels.

\section{Nonlinear Kinetic Equation for a Quantum Subsystem}

The derivation of the kinetic equation (14) shows that interstate transitions in the QS are characterized by the averaged rate constants (16). Since the transitions occur between the states belonging to the whole QS, this equation appears in a linear form. If, however, the QS is associated with the complex system each part of which refers either to a separate subsystem or/and to different degrees of freedom (electronic, nuclear, spin), then the kinetic equation for populations of the subsystem states becomes nonlinear [30].

\subsection{General form of a nonlinear kinetic equation for the subsystem state populations}

We consider an important (in the practical sense) situation where the subsystems are weakly coupled to one another, so that the common QS state $|a\rangle=\left|a_{1} a_{2} \ldots . a_{N}\right\rangle$ ( $N$ is the number of subsystems in the QS) can be represented as a product of separate subsystem states $\left|a_{1}\right\rangle,\left|a_{2}\right\rangle, \ldots$, i.e., $|a\rangle \simeq \prod_{n=1}^{N}\left|a_{n}\right\rangle$. Such a factorization indicates that the off-diagonal interaction $V$ does not strongly perturb the states of separate subsystems. This fact allows one to represent the state probability of the QS as a product

$P(a ; t)=P\left(a_{1} a_{2} \ldots a_{N} ; t\right) \simeq \prod_{n=1}^{N} P\left(a_{n} ; t\right)$, 
where each subsystem state probability $P\left(a_{n} ; t\right)$ satisfies the normalization condition

$\sum_{a_{n}} P\left(a_{n} ; t\right)=1$

From the general point of view, the probability $P\left(a_{n} ; t\right)$ of a realization of the $a_{n}$-th state in the common QS is defined by the expression

$P\left(a_{n} ; t\right)=\sum_{\{a\} \neq a_{n}} P\left(a_{1} a_{2} \ldots a_{N} ; t\right)$

(symbol $\{a\}$ denotes the summation over quantum numbers $\left.a_{1}, a_{2}, \ldots a_{N}\right)$. Therefore, the summation of the leftand right-hand sides of Eq. (14) over all quantum numbers except for $a_{n}$ yields

$$
\dot{P}\left(a_{n} ; t\right)=-\sum_{\{a\} \neq a_{n}} \sum_{\{b\} \neq a_{n}} K\left(a_{1} a_{2} \ldots a_{N} ; b_{1} b_{2} \ldots b_{N}\right) \times
$$$$
\times\left[P\left(a_{n} ; t\right) \prod_{j \neq n} P\left(a_{j} ; t\right)-P\left(b_{n} ; t\right) \prod_{j \neq n} P\left(b_{j} ; t\right)\right] .
$$

Equation (22) is a nonlinear kinetic equation describing the evolution of state probabilities $P\left(a_{n} ; t\right)$ of the $n$-th separate quantum subsystem coupled to another quantum subsystems by the off-diagonal interaction $V^{(t r)}$, Eq. (17). The character of the nonlinearity is dictated by three general factors. The first one is associated with the number of states $a_{1} a_{2}, \ldots a_{N}$ and $b_{1} b_{2} \ldots b_{N}$ involved in the precise $a_{1} a_{2}, \ldots a_{N} \rightleftarrows b_{1} b_{2} \ldots b_{N}$ transition via the transition matrix element $V_{b a}=$ $\left\langle b_{1} b_{2} \ldots b_{N}\left|V^{(t r)}\right| a_{1} a_{2} \ldots a_{N}\right\rangle$. The second factor appears, when certain subsystems in the common QS manifest the properties of macroscopic systems. In this case, the transitions between the microscopic subsystem (that possesses a finite number of degrees of freedom) and the macroscopic system does not change noticeably the state populations of the macroscopic system. The third factor is associated with external sources that are able to support a stationary population of the subsystem levels.

\subsection{Linearization of the kinetic equation}

As an example, let consider the QS that consists of three subsystems $\mathrm{A}, \mathrm{B}$, and $\mathrm{C}$ with respective states $|\alpha\rangle,|\beta\rangle$ and $|\gamma\rangle$. We also assume that the offdiagonal interaction $V$ couples the states of subsystem $\mathrm{B}$ with the states of subsystems $\mathrm{A}$ and $\mathrm{C}$, but does not couple the states of subsystems A and $\mathrm{C}$ (the states $|\alpha\rangle$ and $|\gamma\rangle$ can be only coupled by the second term in Eq. (17), i.e., by the operator $V G(E) V)$. This yields $\left\langle\alpha^{\prime} \beta^{\prime} \gamma^{\prime}\left|V^{(t r)}\right| \alpha \beta \gamma\right\rangle=\delta_{\gamma^{\prime}, \gamma}\left(1-\delta_{\alpha^{\prime} \alpha}\right)(1-$ $\left.\delta_{\beta^{\prime}, \beta}\right)\left\langle\alpha^{\prime} \beta^{\prime}|V| \alpha \beta\right\rangle+\delta_{\alpha^{\prime} \alpha}\left(1-\delta_{\beta^{\prime}, \beta}\right)\left(1-\delta_{\gamma^{\prime}, \gamma}\right)\left\langle\beta^{\prime} \gamma^{\prime}|V| \beta \gamma\right\rangle+$ $\left(1-\delta_{\alpha^{\prime}, \alpha}\right)\left(1-\delta_{\gamma^{\prime}, \gamma}\right)\left\langle\alpha^{\prime} \beta^{\prime} \gamma^{\prime}|V G(E) V| \alpha \beta \gamma\right\rangle$. Bearing this relation in mind, one can derive a nonlinear kinetic equation for each population $P(\xi ; t),(\xi=\alpha, \beta, \gamma)$. Thus, for instance, the time evolution of population $P(\alpha ; t)$ is governed by the kinetic equation

$\dot{P}(\alpha ; t)=-\sum_{\alpha^{\prime} \beta^{\prime}}\left\{\sum_{\beta} K\left(\alpha^{\prime} \beta^{\prime} ; \alpha \beta\right)\left(1-\delta_{\alpha^{\prime}, \alpha}\right)\left(1-\delta_{\beta^{\prime}, \beta}\right) \times\right.$

$\times\left[P(\alpha ; t) P(\beta ; t)-P\left(\alpha^{\prime} ; t\right) P\left(\beta^{\prime} ; t\right)\right]+$

$+\sum_{\beta \gamma \gamma^{\prime}} K\left(\alpha^{\prime} \beta^{\prime} \gamma^{\prime} ; \alpha \beta \gamma\right)\left(1-\delta_{\alpha^{\prime}, \alpha}\right)\left(1-\delta_{\gamma^{\prime}, \gamma}\right) \times$

$\left.\times\left[P(\alpha ; t) P(\beta ; t) P(\gamma ; t)-P\left(\alpha^{\prime} ; t\right) P\left(\beta^{\prime} ; t\right) P\left(\gamma^{\prime} ; t\right)\right]\right\}$.

In Eq. (23), the transfer rate $K\left(\alpha^{\prime} \beta^{\prime} ; \alpha \beta\right)$ is calculated in line with Eq. (16) at $V_{a b}^{(\operatorname{tr})}=\left\langle\alpha^{\prime} \beta^{\prime}|V| \alpha \beta\right\rangle$, $E(a) \simeq E_{\alpha}+E_{\beta}$ and $E(b) \simeq E_{\alpha^{\prime}}+E_{\beta^{\prime}}$. Since only the states belonging to two subsystems are involved in the transitions, the respective term on the right-hand side of the kinetic equation exhibits the second order of nonlinearity. At the same time, the formation of the transfer rate $K\left(\alpha^{\prime} \beta^{\prime} \gamma^{\prime} ; \alpha \beta \gamma\right)$ involves the states belonging to three subsystems in the transition process. This conclusion follows from the analysis of Eq. (16), where now $V_{a b}^{(\mathrm{tr})}=\left\langle\alpha^{\prime} \beta^{\prime} \gamma^{\prime}|V| \alpha \beta \gamma\right\rangle, E(a) \simeq E_{\alpha}+E_{\beta}+E_{\gamma}$ and $E(b) \simeq E_{\alpha^{\prime}}+E_{\beta^{\prime}}+E_{\gamma^{\prime}}$. The given circumstance leads to the third order of nonlinearity in the kinetic equation (Eq. (23) contains the term with the product of three populations).

The kinetic equation (23) reduces its nonlinearity if one or two subsystems possess the macroscopic properties. Let, for instance, $\mathrm{A}$ is the macroscopic subsystem, while B and C are the microscopic ones. Since the transitions between the microscopic and macroscopic subsystems do not noticeably perturb the state populations of the macroscopic subsystem, one can set

$P(\alpha ; t) \simeq W_{A}(\alpha)+\Delta P(\alpha ; t)$,

where the inequality

$\Delta P(\alpha ; t) \ll W_{A}(\alpha)$ 
is assumed to be supported at any time $t$. In Eq. (24), the quantity $W_{A}(\alpha)$ is the equilibrium distribution function for subsystem A. Relation (24) allows one to reduce the nonlinear kinetic equation (23) to the form

$$
\begin{aligned}
& \dot{P}(\alpha ; t)=-\sum_{\alpha^{\prime}}\left(1-\delta_{\alpha^{\prime}, \alpha}\right)\left\{\sum_{\beta \beta^{\prime}}\left(1-\delta_{\beta^{\prime}, \beta}\right) \times\right. \\
& \times\left[K\left(\alpha^{\prime} \beta^{\prime} ; \alpha \beta\right) W_{A}(\alpha)-K\left(\alpha^{\prime} \beta ; \alpha \beta^{\prime}\right) W_{A}\left(E \alpha^{\prime}\right)\right] P(\beta ; t)+ \\
& +\sum_{\beta \beta^{\prime}} \sum_{\gamma \gamma^{\prime}}\left(1-\delta_{\gamma^{\prime}, \gamma}\right)\left[K\left(\alpha^{\prime} \beta^{\prime} \gamma^{\prime} ; \alpha \beta \gamma\right) W_{A}(\alpha)-\right.
\end{aligned}
$$$$
\left.\left.-K\left(\alpha^{\prime} \beta \gamma ; \alpha \beta^{\prime} \gamma^{\prime}\right) W_{A}\left(\alpha^{\prime}\right)\right] P(\beta ; t) P(\gamma ; t)\right\},
$$

which offers a more weak nonlinearity. If the $\mathrm{A}$ and the $\mathrm{C}$ are both macroscopic subsystems, while the $\mathrm{B}$ remains a microscopic subsystem, then the nonlinear kinetic equation reduces to a completely linear one,

$$
\begin{aligned}
& \dot{P}(\alpha ; t)=-\sum_{\alpha^{\prime}}\left(1-\delta_{\alpha^{\prime}, \alpha}\right)\left\{\sum_{\beta \beta^{\prime}}\left(1-\delta_{\beta^{\prime}, \beta}\right) \times\right. \\
& \times\left[K\left(\alpha^{\prime} \beta^{\prime} ; \alpha \beta\right) W_{A}(\alpha)-K\left(\alpha^{\prime} \beta ; \alpha \beta^{\prime}\right) W_{A}\left(\alpha^{\prime}\right)\right] P(\beta ; t)+ \\
& +\sum_{\beta \beta^{\prime}} \sum_{\gamma \gamma^{\prime}}\left(1-\delta_{\gamma^{\prime}, \gamma}\right)\left[K\left(\alpha^{\prime} \beta^{\prime} \gamma^{\prime} ; \alpha \beta \gamma\right) W_{A}(\alpha) W_{C}(\gamma)-\right. \\
& \left.\left.-K\left(\alpha^{\prime} \beta \gamma^{\prime} ; \alpha \beta^{\prime} \gamma\right) W_{A}\left(\alpha^{\prime}\right) W_{C}\left(\gamma^{\prime}\right)\right] P(\beta ; t)\right\}
\end{aligned}
$$

Here, $W_{C}(\gamma)$ is the equilibrium distribution function of subsystem $\mathrm{C}$.

\section{Kinetic Equations for a 1M2 Device}

We consider the transmission of electrons through the 1M2 device under conditions, when the moleculeelectrode couplings do not destroy individual properties of a molecule, so that the molecule can be characterized by its proper states and proper energies. As such, the 1M2 device can be considered as a QS composed from three quantum subsystems, one microscopic subsystem (molecule) and two macroscopic subsystems (electrodes 1 and 2). To employ the results derived in the previous section, let us associate subsystem A with electrode 1 , subsystem B with the molecule and subsystem $\mathrm{C}$ with electrode 2 . We denote the respective quantum states as $\alpha=N_{1 \mathbf{k} \sigma}, \beta=M(\nu)$, and $\gamma=N_{2 \mathbf{k}^{\prime} \sigma^{\prime}}$. Here, $N_{r \mathbf{k} \sigma}=0,1$ is the number of electrons with wave vector $\mathbf{k}$ and spin projection $\sigma$ occupying the conduction band of the $r(=1,2)$-th electrode. The symbol $M(\nu)$ stands for a set of electronic, vibronic and spin quantum numbers of the molecule being in its $\nu(=0, \pm 1, \pm 2, \ldots)$-th charge state. With the use of the given designations, we set

$E(\zeta)=E_{r \mathbf{k} \sigma} N_{r \mathbf{k} \sigma}, \quad(r=1(2)$ if $\zeta=\alpha(\gamma))$

and

$W_{Z}(\zeta)=N_{r \mathbf{k} \sigma} f_{r}\left(E_{r \mathbf{k} \sigma}\right)+\left[1-N_{r \mathbf{k} \sigma}\right]\left[1-f_{r}\left(E_{r \mathbf{k} \sigma}\right)\right]$

$(r=1(2)$ if $Z=A(C), \zeta=\alpha(\gamma))$.

In Eq. (29),

$f_{r}\left(E_{r \mathbf{k} \sigma}\right)=\frac{1}{\exp \left[\left(E_{r \mathbf{k} \sigma}-\mu_{r}\right) / k_{\mathrm{B}} T\right]+1}$

is the Fermi distribution function ( $\mu_{r}$ is the chemical potential for the $r(=1,2)$-th electrode, $k_{\mathrm{B}}$ is the Boltzmann constant, and $T$ is the absolute temperature) of electrons occupying the conduction band of the $r$-th electrode.

\subsection{Kinetic equations and partial transfer rates}

We give a reading form of the kinetic equations for the single-electron probabilities $P(r \mathbf{k} \sigma ; t) \equiv P\left(1_{r \mathbf{k} \sigma} ; t\right)$ (characterizing the occupancy of the conduction band of the $r$-th electrode), as well as for molecular state occupancies $P(M(\nu) ; t)$. The first equation follows from Eq. (27) written for $\alpha=1_{\mathbf{k} \sigma}$ with regard for the inequality $\Delta P(r \mathbf{k} \sigma ; t) \ll f_{r}\left(E_{r \mathbf{k} \sigma}\right)$ (particular case of inequality $(25))$. The desirable kinetic equation reads $\left(r, r^{\prime}=1,2\right)$

$$
\begin{aligned}
& \dot{P}(r \mathbf{k} \sigma ; t)=-\sum_{M M^{\prime}} \sum_{\nu}\left\{\left[K\left(r \mathbf{k} \sigma M(\nu) \rightarrow M^{\prime}(\nu+1)\right)-\right.\right. \\
& \left.-K\left(M(\nu) \rightarrow r \mathbf{k} \sigma M^{\prime}(\nu-1)\right)\right]+ \\
& +\sum_{r^{\prime} \mathbf{k}^{\prime} \sigma^{\prime}(\neq r \mathbf{k} \sigma)}\left[K\left(r \mathbf{k} \sigma, r^{\prime} \mathbf{k}^{\prime} \sigma^{\prime} M(\nu) \rightarrow M^{\prime}(\nu+2)\right)-\right. \\
& \left.-K\left(M(\nu) \rightarrow r \mathbf{k} \sigma, r^{\prime} \mathbf{k}^{\prime} \sigma^{\prime} M^{\prime}(\nu-2)\right)\right]+
\end{aligned}
$$


$+\sum_{\mathbf{k}^{\prime} \sigma^{\prime}}\left(1-\delta_{r^{\prime}, r}\right)\left[K\left(r \mathbf{k} \sigma M(\nu) \rightarrow r^{\prime} \mathbf{k}^{\prime} \sigma^{\prime} M^{\prime}\left(\nu^{\prime}\right)\right)-\right.$

$\left.\left.-K\left(r^{\prime} \mathbf{k} \sigma M(\nu) \rightarrow r \mathbf{k}^{\prime} \sigma^{\prime} M^{\prime}(\nu)\right)\right]\right\} P(M(\nu) ; t)$,

where

$K\left(r \mathbf{k} \sigma M(\nu) \rightarrow M^{\prime}(\nu+1)\right)=\frac{2 \pi}{\hbar}\left|V_{M^{\prime}(\nu+1) ; r \mathbf{k} \sigma M(\nu)}\right|^{2} \times$

$\times f_{r}\left(E_{r \mathbf{k} \sigma}\right) \delta\left[E_{r \mathbf{k} \sigma}+E_{M(\nu)}-E_{M^{\prime}(\nu+1)}\right]$

and

$K\left(M(\nu) \rightarrow r \mathbf{k} \sigma M^{\prime}(\nu-1)\right)=\frac{2 \pi}{\hbar}\left|V_{r \mathbf{k} \sigma M^{\prime}(\nu-1) ; M(\nu)}\right|^{2} \times$

$\times\left[1-f_{r}\left(E_{r \mathbf{k} \sigma}\right)\right] \delta\left[E_{M(\nu)}-E_{r \mathbf{k} \sigma}-E_{M^{\prime}(\nu-1)}\right]$

are the partial (forward and backward, respectively) single-electron transfer rates characterizing the hopping of an electron between the the $r$-th electrode and the molecule. Hereafter, we use the following designation for the transient matrix element:

$V_{M^{\prime}(\nu+1) ; r \mathbf{k} \sigma M(\nu)}=V_{r \mathbf{k} \sigma M(\nu) ; M^{\prime}(\nu+1)}^{*}=$

$=\left\langle 0_{r \mathbf{k} \sigma} M^{\prime}(\nu+1)|V| 1_{r \mathbf{k} \sigma} M(\nu)\right\rangle$.

The two-electron hopping transfer rates are given by the expressions

$K\left(r \mathbf{k} \sigma, r^{\prime} \mathbf{k}^{\prime} \sigma^{\prime} M(\nu) \rightarrow M^{\prime}(\nu+2)\right)=$

$=\frac{2 \pi}{\hbar} f_{r}\left(E_{r \mathbf{k} \sigma}\right) f_{r^{\prime}}\left(E_{r^{\prime} \mathbf{k}^{\prime} \sigma^{\prime}}\right) \times$

$\times\left|\sum_{\tilde{M}} \frac{V_{M^{\prime}(\nu+2) ; r^{\prime} \mathbf{k}^{\prime} \sigma^{\prime} \tilde{M}(\nu+1)} V_{\tilde{M}(\nu+1) ; r \mathbf{k} \sigma M(\nu)}}{E_{r \mathbf{k} \sigma}+\mathcal{E}(M(\nu))-\mathcal{E}(\tilde{M}(\nu+1))}\right|^{2} \times$

$\times \delta\left[E_{r \mathbf{k} \sigma}+E_{r^{\prime} \mathbf{k}^{\prime} \sigma^{\prime}}+E_{M(\nu)}-E_{M^{\prime}(\nu+2)}\right]$

and

$K\left(M(\nu) \rightarrow r \mathbf{k} \sigma, r^{\prime} \mathbf{k}^{\prime} \sigma^{\prime} M^{\prime}(\nu-2)\right)=$

$=\frac{2 \pi}{\hbar}\left[1-f_{r}\left(E_{r \mathbf{k} \sigma}\right)\right]\left[1-f_{r^{\prime}}\left(E_{r^{\prime} \mathbf{k}^{\prime} \sigma^{\prime}}\right)\right] \times$
$\times\left|\sum_{\tilde{M}} \frac{V_{r^{\prime} \mathbf{k}^{\prime} \sigma^{\prime} M^{\prime}(\nu-2) ; \tilde{M}(\nu-1)} V_{r \mathbf{k} \sigma \tilde{M}(\nu-1) ; M(\nu)}}{E_{r \mathbf{k} \sigma}+\mathcal{E}(\tilde{M}(\nu-1)-\mathcal{E}(M(\nu)))}\right|^{2} \times$

$\times \delta\left[E_{M(\nu)}-E_{r \mathbf{k} \sigma}-E_{r^{\prime} \mathbf{k}^{\prime} \sigma^{\prime}}-E_{M^{\prime}(\nu-2)}\right]$.

(At $r=r^{\prime}$, one has only to remain the terms, where $\left.\mathbf{k}^{\prime} \sigma^{\prime} \neq \mathbf{k} \sigma\right)$.

The above-given single-electron and two-electron partial transfer rates characterize the transmission processes accompanied by a molecular recharge. At the same time, the kinetics of charge transfer includes the processes at which an electron transmission does not bring to the molecular charging but initiate molecular transitions $M(\nu) \rightleftarrows M^{\prime}(\nu)$. The respective transfer rate reads $\left(r^{\prime} \neq r\right)$

$K\left(r \mathbf{k} \sigma M(\nu) \rightarrow r^{\prime} \mathbf{k}^{\prime} \sigma^{\prime} M^{\prime}(\nu)\right)=$

$=\frac{2 \pi}{\hbar} f_{r}\left(E_{r \mathbf{k} \sigma}\right)\left[1-f_{r^{\prime}}\left(E_{r^{\prime} \mathbf{k}^{\prime} \sigma^{\prime}}\right)\right] \times$

$\times \mid \sum_{\tilde{M}}\left[\frac{V_{M^{\prime}(\nu) ; r^{\prime} \mathbf{k}^{\prime} \sigma^{\prime} \tilde{M}(\nu-1)} V_{r \mathbf{k} \sigma \tilde{M}(\nu-1) ; M(\nu)}}{\mathcal{E}(M(\nu))-E_{r^{\prime} \mathbf{k}^{\prime} \sigma^{\prime}}-\mathcal{E}(\tilde{M}(\nu-1))}+\right.$

$\left.+\frac{V_{r^{\prime} \mathbf{k}^{\prime} \sigma^{\prime} M^{\prime}(\nu) ; \tilde{M}(\nu+1)} V_{\tilde{M}(\nu+1) ; r \mathbf{k} \sigma M(\nu)}}{E_{r \mathbf{k} \sigma}+\mathcal{E}(M(\nu))-\mathcal{E}(\tilde{M}(\nu+1))}\right]\left.\right|^{2} \times$

$\times \delta\left[E_{r \mathbf{k} \sigma}+E_{M(\nu)}-E_{r^{\prime} \mathbf{k}^{\prime} \sigma^{\prime}}-E_{M^{\prime}(\nu)}\right]$.

In Eqs. (35)-(37), the quantity

$\mathcal{E}(M(\nu)) \simeq E_{M(\nu)}+\Sigma_{M(\nu)}^{(1)}(\mathcal{E})+\Sigma_{M(\nu)}^{(2)}(\mathcal{E})$

is the modified molecular energy that contains selfenergies $(r=1,2)$

$\Sigma_{M(\nu)}^{(r)}(\mathcal{E}) \simeq \sum_{\mathbf{k} \sigma} \sum_{M^{\prime}} \frac{\left|V_{M(\nu) ; r \mathbf{k} \sigma M^{\prime}(\nu-1)}\right|^{2}}{\mathcal{E}-\left(E_{r \mathbf{k} \sigma}+E_{M^{\prime}(\nu-1)}\right)+i 0^{+}}$.

The appearance of the self-energy is caused by the interaction of the molecule with the electrodes [40]. The imaginary part of the self-energy,

$\Gamma_{M(\nu)}^{(r)}(\mathcal{E})=$

$=\sum_{M^{\prime}} \Gamma^{(r)}\left(M^{\prime}(\nu-1) \rightarrow M(\nu) ; \mathcal{E}-E_{M^{\prime}(\nu-1)}\right)$, 
defines the broadening of molecular levels through the transition width parameters

$\Gamma^{(r)}\left(M^{\prime}(\nu-1) \rightarrow M(\nu) ; \mathcal{E}-E_{M^{\prime}(\nu-1)}\right) \simeq 2 \pi \times$

$\times \sum_{\mathbf{k} \sigma}\left|V_{M(\nu) ; r \mathbf{k} \sigma M^{\prime}(\nu-1)}\right|^{2} \delta\left[\left(\mathcal{E}-E_{M^{\prime}(\nu-1)}\right)-E_{r \mathbf{k} \sigma}\right]$.

The kinetic equation for molecular state occupancies is derived analogously. It reads

$$
\begin{aligned}
& \dot{P}(M(\nu) ; t)=-\sum_{M^{\prime}} \sum_{r \mathbf{k} \sigma}\left\{\left[K\left(r \mathbf{k} \sigma M(\nu) \rightarrow M^{\prime}(\nu+1)\right)+\right.\right. \\
& \left.+K\left(M(\nu) \rightarrow r \mathbf{k} \sigma M^{\prime}(\nu-1)\right)\right] P(M(\nu) ; t)- \\
& -\left[K\left(M^{\prime}(\nu+1) \rightarrow r \mathbf{k} \sigma M(\nu)\right) P\left(M^{\prime}(\nu+1) ; t\right)+\right. \\
& \left.\left.+K\left(r \mathbf{k} \sigma M^{\prime}(\nu-1) \rightarrow M(\nu)\right) P\left(M^{\prime}(\nu-1) ; t\right)\right]\right\}- \\
& -\sum_{M^{\prime}} \sum_{r \mathbf{k} \sigma} \sum_{r^{\prime} \mathbf{k}^{\prime} \sigma^{\prime}}\left(1-\delta_{r^{\prime} \mathbf{k}^{\prime} \sigma^{\prime}, r \mathbf{k} \sigma}\right) \times
\end{aligned}
$$$$
\times\left\{\left[K\left(r \mathbf{k} \sigma, r^{\prime} \mathbf{k}^{\prime} \sigma^{\prime} M(\nu) \rightarrow M^{\prime}(\nu+2)\right)+\right.\right.
$$$$
\left.+K\left(M(\nu) \rightarrow r \mathbf{k} \sigma, r^{\prime} \mathbf{k}^{\prime} \sigma^{\prime} M^{\prime}(\nu-2)\right)\right] P(M(\nu) ; t)-
$$$$
-\left[K\left(M^{\prime}(\nu+2) \rightarrow r \mathbf{k} \sigma, r^{\prime} \mathbf{k}^{\prime} \sigma^{\prime} M(\nu)\right) P\left(M^{\prime}(\nu+2) ; t\right)+\right.
$$$$
\left.+K\left(r \mathbf{k} \sigma, r^{\prime} \mathbf{k}^{\prime} \sigma^{\prime} M^{\prime}(\nu-2) \rightarrow M(\nu)\right) P\left(M^{\prime}(\nu-2) ; t\right)\right]+
$$$$
+\sum_{r^{\prime}} \sum_{r \mathbf{k} \sigma} \sum_{r^{\prime} \mathbf{k}^{\prime} \sigma^{\prime}}\left[K\left(r \mathbf{k} \sigma M(\nu) \rightarrow r^{\prime} \mathbf{k}^{\prime} \sigma^{\prime} M^{\prime}(\nu)\right) P(M(\nu) ; t)-\right.
$$$$
\left.\left.-K\left(r^{\prime} \mathbf{k} \sigma M(\nu) \rightarrow r \mathbf{k}^{\prime} \sigma^{\prime} M^{\prime}(\nu)\right) P\left(M^{\prime}(\nu) ; t\right)\right]\right\}-
$$$$
-\sum_{M^{\prime}} \sum_{r \mathbf{k} \sigma}\left[K\left(r \mathbf{k} \sigma M(\nu) \rightarrow r \mathbf{k} \sigma M^{\prime}(\nu)\right) P(M(\nu) ; t)-\right.
$$$$
\left.-K\left(r \mathbf{k} \sigma M^{\prime}(\nu) \rightarrow r \mathbf{k} \sigma M(\nu)\right) P\left(M^{\prime}(\nu) ; t\right)\right] .
$$

Here, all charge transfer rates are defined by Eqs. (32)(37) except the transfer rate

$$
K\left(r \mathbf{k} \sigma M(\nu) \rightarrow r \mathbf{k} \sigma M^{\prime}(\nu)\right)=
$$

$=\frac{2 \pi}{\hbar}\left\{f_{r}\left(E_{r \mathbf{k} \sigma}\right)\left|\sum_{\tilde{M}} \frac{V_{r \mathbf{k} \sigma M^{\prime}(\nu) ; \tilde{M}(\nu+1)} V_{\tilde{M}(\nu+1) ; r \mathbf{k} \sigma M(\nu)}}{E_{r \mathbf{k} \sigma}+\mathcal{E}(M(\nu))-\mathcal{E}(\tilde{M}(\nu+1))}\right|^{2}+\right.$ $\left.+\left(1-f_{r}\left(E_{r \mathbf{k} \sigma}\right)\right)\left|\sum_{\tilde{M}} \frac{V_{M^{\prime}(\nu) ; r \mathbf{k} \sigma \tilde{M}(\nu-1)} V_{r \mathbf{k} \sigma \tilde{M}(\nu-1) ; M(\nu)}}{\mathcal{E}(M(\nu))-E_{r \mathbf{k} \sigma}-\mathcal{E}(\tilde{M}(\nu-1))}\right|^{2}\right\} \times$

$\times \delta\left[E_{M(\nu)}-E_{M^{\prime}(\nu)}\right]$.

This rate characterizes an electron transition within the $1 \mathrm{M} 2$ device when the transmission is not accompanied by a molecular recharge.

\subsection{Currents in the 1M2 device}

In the 1M2 device, the current from the $r$-th electrode to the attached molecule is defined as

$I_{r}(t)=e \dot{N}_{r}(t)$

where $e=-|e|$ is the electron charge, and $N_{r}(t)$ is the number of electrons in the conduction band of the $r$-th electrode. By virtue of the fact that $\dot{N}_{r}(t)=$ $\sum_{\mathbf{k} \sigma} \dot{P}(r \mathbf{k} \sigma ; t)$, one derives the expression for the current as the sum of two components,

$I_{r}(t)=I_{\mathrm{seq}}^{(r)}(t)+I_{\mathrm{dir}}^{(r)}(t)$.

The first component,

$$
\begin{aligned}
& I_{\mathrm{seq}}^{(r)}(t)=I_{0} \pi \hbar \sum_{M M^{\prime}} \sum_{\nu}\left\{\left[K\left(r M(\nu) \rightarrow M^{\prime}(\nu+1)\right)-\right.\right. \\
& \left.-K\left(M(\nu) \rightarrow r M^{\prime}(\nu-1)\right)\right]+\left[K\left(r r^{\prime} M(\nu) \rightarrow M^{\prime}(\nu+2)\right)-\right. \\
& \left.\left.-K\left(M(\nu) \rightarrow r r^{\prime} M^{\prime}(\nu-2)\right)\right]\right\} P(M(\nu) ; t)
\end{aligned}
$$

is formed owing to the single-electron and two-electron hopping processes in the 1M2 device (the first and second terms on the right-hand side of Eq. (46), respectively). The second current component,

$$
\begin{aligned}
& I_{d i r}^{(r)}(t)=I_{0} \pi \hbar \sum_{M M^{\prime}} \sum_{\nu}\left[K\left(r M(\nu) \rightarrow r^{\prime} M^{\prime}(\nu)\right)-\right. \\
& \left.-K\left(r^{\prime} M(\nu) \rightarrow r M^{\prime}(\nu)\right)\right]\left(1-\delta_{r, r^{\prime}}\right) P(M(\nu) ; t),
\end{aligned}
$$

reflects the presence of the direct electron transmission from one electrode to another. (In Eqs. (46) and (47), $I_{0}=|e| / \pi \hbar \approx 77.6 \mu \mathrm{A}$ is the current unit [3]). 
It follows from Eqs. (46) and (47) that the time behavior of the current is completely determined by the evolution of molecular state probabilities. In accord with Eq. (42), this evolution is described by the kinetic equation

$$
\begin{aligned}
& \dot{P}(M(\nu) ; t)=-\sum_{M^{\prime}} \sum_{r}\left\{\left[K\left(r M(\nu) \rightarrow M^{\prime}(\nu+1)\right)+\right.\right. \\
& +K\left(M(\nu) \rightarrow r M^{\prime}(\nu-1)\right)+ \\
& +\sum_{r^{\prime}}\left[K\left(r r^{\prime} M(\nu) \rightarrow M^{\prime}(\nu+2)\right)+\right. \\
& +K\left(M(\nu) \rightarrow r r^{\prime} M^{\prime}(\nu-2)\right)+ \\
& \left.\left.+K\left(r M(\nu) \rightarrow r^{\prime} M^{\prime}(\nu)\right)\right]\right] P(M(\nu) ; t)- \\
& -\left[K\left(M^{\prime}(\nu+1) \rightarrow r M(\nu)\right) P\left(M^{\prime}(\nu+1) ; t\right)+\right. \\
& +K\left(r M^{\prime}(\nu-1) \rightarrow M(\nu)\right) P\left(M^{\prime}(\nu-1) ; t\right)+ \\
& +\sum_{r^{\prime}}\left[K\left(M^{\prime}(\nu+2) \rightarrow r r^{\prime} M(\nu)\right) P\left(M^{\prime}(\nu+2) ; t\right)+\right. \\
& +K\left(r r^{\prime} M^{\prime}(\nu-2) \rightarrow M(\nu)\right) P(M(\nu-2) ; t)+ \\
& \left.\left.\left.+K\left(r^{\prime} M^{\prime}(\nu) \rightarrow r M(\nu)\right)\right] P\left(M^{\prime}(\nu) ; t\right)\right]\right\}
\end{aligned}
$$

\subsection{Rate constants}

It follows from expressions (46), (47), and (48) that, within the kinetic approach, the current formation and the transitions between molecular states are characterized by the common rate constants. The latter are calculated as the sum (over single-electron band states $\mathbf{k} \sigma$ and $\mathbf{k}^{\prime} \sigma^{\prime}$ ) of above partial transfer rates. The form of rate constants is determined by transmission processes in the precise 1M2 device. Nevertheless, in certain cases, one can derive a rather simple reading form for the rate constants. As an example, we consider two of them.

Contact rate constants. The forward contact rate constant characterizes the hopping of an electron from the $r$-th electrode to the molecule. It is determined by the expression $K\left(r M(\nu) \rightarrow M^{\prime}(\nu+1)\right)=$ $\sum_{r \mathbf{k} \sigma} K\left(r \mathbf{k} \sigma M(\nu) \rightarrow M^{\prime}(\nu+1)\right)$, which can be represented in the form

$K\left(r M(\nu) \rightarrow M^{\prime}(\nu+1)\right)=\frac{1}{\hbar} \Gamma^{(r)}\left(M(\nu) \rightarrow M^{\prime}(\nu+1) ; E\right) \times$

$\times N\left(\Delta E_{M^{\prime}(\nu+1), M(\nu)}^{(r)}\right)$.

Here, the first multiplier is defined by Eq. (41) at $\mathcal{E}=$ $E_{M^{\prime}(\nu+1)}-E_{N(\nu)}$, whereas

$N\left(\Delta E_{M^{\prime}(\nu+1), M(\nu)}^{(r)}\right)=\left\{\exp \left[\frac{\Delta E_{M^{\prime}(\nu+1), M(\nu)}^{(r)}}{k_{\mathrm{B}} T}\right]+1\right\}^{-1}$

is the distribution function with

$\Delta E_{M^{\prime}(\nu+1), M(\nu)}^{(r)}=E_{M^{\prime}(\nu+1)}^{(r)}-\left(E_{M(\nu)}^{(r)}+\mu_{r}\right)$

being the transmission gap for the $r M(\nu) \rightarrow M^{\prime}(\nu+1)$ charge transfer route $\left(\mu_{r}\right.$ is the chemical potential of the $r$-th electrode). The expression for the backward contact rate constant, $K\left(M^{\prime}(\nu+1) \rightarrow r M(\nu)\right)$, follows from Eq. (49), if one substitutes $N\left(\Delta E_{M^{\prime}(\nu+1), M(\nu)}^{(r)}\right)$ for $\left(1-N\left(\Delta E_{M^{\prime}(\nu+1), M(\nu)}^{(r)}\right)\right.$. In the absence of magnetic interactions, a spin state of the molecule and a spin projection of the transferred electron are good quantum numbers. Therefore, to specify a molecular state, one has to indicate a molecular term along with respective vibration states. Let, for instance, the ground state of the molecule has zero charge $(\nu=0)$ and zero spin $(S=0)$. In the Condon approximation, the noted state can be indicated as $M(0)=M_{0} v_{0}$ ( $v_{0}$ is the set of vibrational quantum numbers in the ground molecular state). When the molecule captures an extra (transferred) electron, it becomes negative. Indicating this fact by sign "-", we set $M^{\prime}(1)=M_{-} v_{-}$. This yields $V_{M^{\prime}(\nu+1) ; r \mathbf{k} \sigma M(\nu)}=V_{M_{-} ; r \mathbf{k} M_{0}}\left\langle v_{-} \mid v_{0}\right\rangle$, where $V_{M_{-} ; r \mathbf{k} M_{0}}$ is the pure electronic matrix element, and $\left\langle v_{-} \mid v_{0}\right\rangle$ is the overlap integral for nuclear wave functions. Thus, in the Condon approximation, $\Gamma^{(r)}\left(M(\nu) \rightarrow M^{\prime}(\nu+1) ; E\right)=$ $\Gamma_{M_{0} \rightarrow M_{-}}^{(r)}(E)\left\langle v_{-} \mid v_{0}\right\rangle^{2}$. The form for $\Gamma_{M_{0} \rightarrow M_{-}}^{(r)}(E)$ can be simplified if one employs the wide-band approximation $[3,8]$, whereby the width parameter could be chosen as energy-independent. If the $M_{0} \rightarrow M_{-}$transition is associated with the appearance of an extra (transferred) electron at the $\lambda$ th molecular orbital, then the quantity $\Gamma_{\lambda}^{(r)} \equiv \Gamma_{M_{0} \rightarrow M_{-}}^{(r)}(E)$ can be considered as the device parameter. As a result, the rate constant for the $r M_{0} \rightarrow M^{\prime}(1)$ transition reduces to the simple form

$K\left(r M(0) \rightarrow M^{\prime}(1)\right)=\frac{1}{\hbar} \Gamma_{\lambda}^{(r)}\left\langle v_{-} \mid v_{0}\right\rangle^{2} \times$ 
$\times N\left(\Delta E_{M_{-} v_{-}, M_{0} v_{0}}^{(r)}\right)$,

where

$\Delta E_{M_{-} v_{-}, M_{0} v_{0}}^{(r)}=\Delta E_{-0}^{(r)}+\hbar\left(\omega_{-} v_{-}-\omega_{0} v_{0}\right)$.

In Eq. (53), the quantities $\omega_{-}$and $\omega_{0}$ indicate the vibrational modes for respective molecular terms, whereas

$\Delta E_{-0}^{(r)}=E_{-}-\left(E_{0}+\mu_{r}\right)$

is the pure electronic transmission gap. [The lowest energies $E_{-}$and $E_{0}$ of the term include the energies $\hbar \omega_{-} / 2$ and $\hbar \omega_{0} / 2$ of respective zero-point vibrations]. In the Holstein model, the frequencies of vibrational modes do not depend on electronic state of a molecule so that $\omega_{-}=\omega_{0}$. At negligible nuclear displacements, one can also set $\left\langle v_{-} \mid v_{0}\right\rangle \approx \delta_{v_{-}, v_{0}}$. Thus, the simplest form for the contact rate constant reads

$K\left(r M(0) \rightarrow M^{\prime}(1)\right)=\frac{1}{\hbar} \Gamma_{\lambda}^{(r)} \delta_{v_{-}, v_{0}} N\left(\Delta E_{-0}^{(r)}\right)$

Distant rate constants. These rate constants characterize two types of transitions. One type reflects twoelectron hopping processes at which two electrons belonging to different electrodes charge the molecule (respective rate constant is $K\left(r r^{\prime} M(\nu) \rightarrow M^{\prime}(\nu+2)\right)$ ) or the molecule returns two electrons to different electrodes (respective rate constant is $K\left(M^{\prime}(\nu+2) \rightarrow r r^{\prime} M(\nu)\right)$ ). The second type of rate constants characterizes the distant single-electron one-step transition process at which an electron is transferred from one electrode to another one. During such a process, the molecule can conserve or change its electronic state, but the molecular charge is not varied (respective rate constant is $\left.K\left(r M(\nu) \rightarrow r^{\prime} M^{\prime}(\nu)\right)\right)$. As an example, we give a reading form for the rate constants characterizing the $1 M(0) \rightarrow 2 M^{\prime}(0)$ inter-electrode electron transfer at $M^{\prime}=M$ and $M^{\prime}=M^{*}$, where $M^{*}$ denotes the excited state of the molecule. The desirable rate constant is estimated in line with the expression $K(r M(\nu) \rightarrow$ $\left.r^{\prime} M^{\prime}(\nu)\right)=\sum_{r \mathbf{k} \sigma} \sum_{r^{\prime} \mathbf{k}^{\prime} \sigma^{\prime}} K\left(r \mathbf{k} \sigma M(\nu) \rightarrow r^{\prime} \mathbf{k}^{\prime} \sigma^{\prime} M^{\prime}(\nu)\right)$ at $\nu=0, r=1, r^{\prime}=2$. It follows from Eq. (37) that the intermediate molecular states $\tilde{M}$ refer either to the negatively or positively charged molecule. In the first case, the intermediate states are associated with the molecule, where an extra electron occupies one of the lowest unoccupied molecular orbitals (LUMOs). In the second case, one electron is absent at one of the highest occupied molecular orbitals (HOMOs). The model under consideration supposes a negligible nuclear displacement during the transmission, as well as the participation of only two frontier MOs, one LUMO, and one HOMO (LUMOHOMO model) in the transfer process. As a result, the summation over the $\tilde{M}$ is removed, and one can introduce the width parameters $\Gamma_{\mathrm{L}}^{(r)}$ and $\Gamma_{\mathrm{H}}^{(r)}$ associated with the LUMO (L) and HOMO $(\mathrm{H})$. The calculations give

$K(1 M(0) \rightarrow 2 M(0))=\frac{2}{\pi \hbar} \times$

$\times\left\{\frac{\Gamma_{\mathrm{L}}^{(1)} \Gamma_{\mathrm{L}}^{(2)}}{\Gamma_{\mathrm{L}}}\left[\operatorname{arctg} \frac{2 \Delta E_{-0}^{(2)}}{\Gamma_{\mathrm{L}}}-\operatorname{arctg} \frac{2 \Delta E_{-0}^{(1)}}{\Gamma_{\mathrm{L}}}\right]+\right.$

$+\frac{\Gamma_{\mathrm{H}}^{(1)} \Gamma_{\mathrm{H}}^{(2)}}{\Gamma_{\mathrm{H}}}\left[\operatorname{arctg} \frac{2 \Delta E_{+0}^{(1)}}{\Gamma_{\mathrm{H}}}-\operatorname{arctg} \frac{2 \Delta E_{+0}^{(2)}}{\Gamma_{\mathrm{H}}}\right]$,

where $\Gamma_{\lambda}=\Gamma_{\lambda}^{(1)}+\Gamma_{\lambda}^{(2)},(\lambda=\mathrm{L}, \mathrm{H})$. The transmission gaps $\Delta E_{-0}^{(1)}$ and $\Delta E_{-0}^{(2)}$ are given by Eq. (54), while transmission gaps $\Delta E_{+0}^{(1)}$ and $\Delta E_{+0}^{(2)} \operatorname{read}(r=1,2)$

$\Delta E_{+0}^{(r)}=\left(E_{+}+\mu_{r}\right)-E_{0}$

Here, $E_{+}$is the lowest energy of the electronic term belonging to the oxidized molecule.

The appearance of molecular state $M^{*}$ is associated with the transition of an intramolecular electron from the HOMO to the LUMO. In the framework of the LUMO-HOMO model, one derives

$K\left(1 M(0) \rightarrow 2 M^{*}(0)\right)=\frac{1}{\pi \hbar} \times$

$\times\left\{\frac{\Gamma_{\mathrm{L}}^{(1)} \Gamma_{\mathrm{H}}^{(2)}}{\Gamma_{\mathrm{L}}}\left[\operatorname{arctg} \frac{2 \Delta E_{-*}^{(2)}}{\Gamma_{\mathrm{L}}}-\operatorname{arctg} \frac{2 \Delta E_{-0}^{(1)}}{\Gamma_{\mathrm{L}}}\right]+\right.$

$+\frac{\Gamma_{\mathrm{L}}^{(1)} \Gamma_{\mathrm{H}}^{(2)}}{\Gamma_{\mathrm{H}}}\left[\operatorname{arctg} \frac{2 \Delta E_{+*}^{(1)}}{\Gamma_{\mathrm{H}}}-\operatorname{arctg} \frac{2 \Delta E_{+0}^{(2)}}{\Gamma_{\mathrm{H}}}\right]$,

where

$\Delta E_{-*}^{(r)}=E_{-}-\left(E_{*}+\mu_{r}\right)$

and

$\Delta E_{+*}^{(r)}=\left(E_{+}+\mu_{r}\right)-E_{*}$

are the transmission gaps associated with the presence of the excited molecular state $\left(E_{*}\right.$ is the lowest energy of an electronic term belonging to the excited molecule). 


\section{Conclusion}

In the present paper, the method of nonequilibrium density matrix is used to derive a generalized master equation for the averaged (over the fast stochastic field) state probabilities of the quantum system, Eq. (14). Under conditions of a weak interaction between the subsystems forming the common quantum system, the nonlinear kinetic equation (22) has been derived for a separate subsystem. The nonlinear kinetic equation is transformed into a linear one, if the microscopic subsystem interacts with macroscopic subsystems. This general result has been demonstrated by the example of the transformation of the nonlinear kinetic equation (23) into the linear balance-like equation (27). Associating two macroscopic subsystems with the electrodes and a microscopic subsystem with the molecule, we deduce the kinetic equation for the band state probabilities, Eq. (31), and specify the form of the respective transfer rates (35)-(37). The application of the density matrix approach to the description of the charge transmission in the 1M2 device allows us to derive the expressions for the sequential (hopping) and direct (distant) components of the current (cf. respective equations (46) and (47)). Additionally, we have at hand the closed system of kinetic equations for molecular state probabilities, Eq. (48). [In separate cases, one can derive rather simple analytic expressions for the contact and distant rate constants, cf. Eqs. (52), (56), and (58).] The general expressions derived for the current and the state occupancies, can be applied to the description of various regimes of current formation in various molecular junctions including those with a magnetic ordering.

The work was supported by special program "Fundamental Properties of Physical Systems under External Conditions" of the Nat. Acad. Sci. of Ukraine.

1. Molecular Electronics, edited by M. Ratner and J. Jortner (Blackwell Science, Oxford, 1997).

2. R.M. Metzger, Acc. Chem. Res. 32, 950 (1999).

3. A. Nitzan, Annu. Rev. Phys. Chem. 52, 681 (2001).

4. P. Hänggi, M. Ratner, and S. Yaliraki, Chem. Phys. 281, 111 (2002).

5. A. Nitzan and M. Ratner, Science 300, 1384 (2003).

6. S. Datta, Nanotechnology 15, S483 (2004).

7. Introducing Molecular Electronics, edited by G. Cuniberti, G.F. Fagas, and K. Richter, Lecture Notes in Physics 680 (Springer, Heidelberg, 2005).

8. M. Galperin, M.A. Ratner, and A. Nitzan, J. Phys. Condens. Matter 19, 103201 (2007).
9. P. Damle, A.W. Ghosh, and S. Datta, Chem. Phys. 281, 171 (2002).

10. G. Vignale and M. Di Ventra, Phys. Rev. B 79, 014201 (2009).

11. S. Nakajima, Progr. Theor. Phys. 20, 948 (1958).

12. R. Zwanzig, Physica 30, 1109 (1964).

13. P.N. Argyres and P.L. Kelley, Phys. Rev. 134, A97 (1964).

14. D.N. Zubarev, Nonequilibrium Statistical Thermodynamics (Consultants Bureau, New York, 1974).

15. A.I. Akhiezer and S.V. Peletminsky, Methods of Statistical Physics (Pergamon Press, New York, 1981).

16. V.P. Seminozhenko, Phys. Reports 91, 104 (1982).

17. E.G. Petrov, Physics of Charge Transfer in Biosystems (Kiev, Naukova Dumka, 1984) (in Russian).

18. K. Blum, Density Matrix Theory and Applications (Plenum Press, New York, 1996).

19. V. May and O. Kühn, Charge and Energy Transfer Dynamics in Molecular Systems (Wiley-VCH, Weinheim, 2004).

20. E.G. Petrov and P. Hänggi, Phys. Rev. Lett. 86, 2862 (2001).

21. J. Lehmann, G.L. Ingold, and P. Hänggi, Chem. Phys. 281, 199 (2002).

22. E.G. Petrov, V. May, and P. Hänggi, Chem. Phys. 319, 380 (2005).

23. E.G. Petrov, Low Temp. Phys. 31, No. 3-4, 338 (2005).

24. E.G. Petrov, Chem. Phys. 326, 151 (2006).

25. E.G. Petrov, V. May, and P. Hänggi, Phys. Rev. B 73, 045498 (2006).

26. F.J. Kaiser, M. Strass, S. Kohler, and P. Hänggi, Chem. Phys. 322, 193 (2006).

27. E.G. Petrov, in Electron Correlation in New Materials and Nanosystems, edited by K. Scharnberg and S. Kruchinin, NATO Science Series II. Mathematics, Physics, and Chemistry, 241, (Kluwer, Dordrecht, 2007), p. 37.

28. R. Jorn and T. Seideman, J. Chem. Phys. 129, 194703 (2008).

29. E.G. Petrov and M.V. Koval, Phys. Lett. A 20, 948 (2008).

30. E.G. Petrov, Ukr. J. Phys. 55, 12 (2010)

31. E.G. Petrov and V.I. Teslenko, Chem. Phys. 375, 243 (2010).

32. V.E. Shapiro and M.N. Loginov, Physica A 91, 563 (1978).

33. E.G. Petrov and V.I. Teslenko, Theor. Math. Phys. 84, 986 (1990).

34. I.A. Goychuk, E.G. Petrov and V. May, Phys. Rev. E 56, 1421 (1997). 
35. E.G. Petrov, Phys. Rev. E 57, 94 (1998).

36. I. Goychuk and P. Hänggi, Adv. Phys. 54, 525 (2005).

37. A more exact form for the transfer rate (14) characterizing the quasiisoenergetic transitions in an open QS, has been derived in [31]. The rate contains a specific Lorentzian. Note, however, that the area covered by this Lorentzian is mainly concentrated near $E(a) \approx E(b)$. Therefore, it becomes possible to simplify the form of the transfer rate $K(a ; b)$ using the delta-function instead of the Lorentzian (see also [30]). The advantage of such a substitution is in that the transfer rate can be calculated without specification of the stochastic field alternating the level positions.

38. After finishing the averaged procedure, the characteristics of a stochastic field are concentrated in the specific Lorentzian [37].

39. A.S. Davydov, Quantum Mechanics (Pergamon Press, Oxford, 1976).

40. Quantity (38) is the proper energy with the modified molecular Hamiltonian $H^{(\mathrm{eff})}$. Its form follows from the diagonalization of the entire Hamiltonian (18) under the condition that the molecule-electrode couplings do not modify the band levels of the macroscopic electrodes (see $[3,8,9,23,24]$ for more details).

Received 01.03.11
КІНЕТИКА ЗАРЯДОВО-ТРАНСПОРТНИХ

ПРОЦЕСІВ У МОЛЕКУЛЯРНИХ З'ЄДНАННЯХ

Е.Г. Петров

Р е $з$ ю м е

Отримано керуюче кінетичне рівняння для ймовірностей реалізації станів квантової системи, що складається з окремих квантових підсистем. Рівняння дозволяє проводити опис процесів транспорту зарядів у молекулярних з'єднаннях, в яких молекула, що вбудована між електродами, виконує роль трансмітера електронів. Особливу увагу приділено отриманню виразів для контактних та дистанційних констант швидкостей, що відповідають за формування послідовної (стрибкової) та прямої (тунельної) компонент струму, а також за часову еволюцію ймовірностей реалізації молекулярних станів. 